\title{
EDITORIAL
}

\section{Steering for success}

\author{
An FDA initiative to enable companies to discuss options for future trial design following \\ completion of early-stage trials could have an important role in addressing the high costs \\ and failure rates of pivotal clinical trials.
}

During drug development, sponsors and regulatory agencies such as the FDA participate in meetings at which the development strategy is discussed, considering factors such as the design of clinical trials. These meetings, which can occur at several stages in the process, are generally considered valuable by sponsors. However, in the past, they have not typically allowed for detailed discussion of the use of novel tools such as modelling and simulation - for example, to aid dose selection prior to pivotal trials.

The potential importance of meetings between sponsors and regulators before pivotal trials are initiated has been indicated by several studies. For example, in 2006, an independent study of the FDA to investigate the factors affecting the likelihood that regulatory applications are approved in the first 'cycle' of FDA review was reported ${ }^{1}$. Among the most important findings of the study, which analysed 63 new drug applications (NDAs) and 14 biologic licence applications (BLAs) from 2002-2004, was the positive impact of meetings between sponsors and the FDA at the end of Phase II trials. For the 46 products for which such meetings were held, 52\% received first-cycle approval, compared with $29 \%$ of products for which such meetings did not occur. Although also important, meetings at the pre-NDA/BLA stage seemed to be less beneficial; products with such meetings had a first-cycle success rate of $47 \%$, compared with $33 \%$ for those without such meetings.

The FDA itself has also been investigating the potential value of meetings before pivotal trials as part of a pilot programme that began in 2004 . Through this programme, the FDA conducted a series of meetings with sponsors at the end of Phase IIa trials, in which information from prior clinical trials, such as dose-response, placebo effect levels and baseline data, was used to derive models and conduct simulations to evaluate the proposed trial design and alternatives, to maximize the chances of success.

For example, in one reported case study of a drug in development for insomnia, the sponsor was seeking to identify the optimal dose range and duration of Phase IIb trials on the basis of studies in healthy subjects ${ }^{2}$. Data derived from published trials of another insomnia drug, as well as data from two internal FDA submissions
- comprising 14 previous trials in total - were used to help understand the quantitative relationship between drug effects in healthy subjects and in patients with insomnia. The sponsor reported that the FDA input led to a redesign of the later trials ${ }^{2}$.

Following on from the pilot programme, the FDA has now issued guidance on meetings at the end of Phase IIa trials $^{3}$. According to this guidance, the main objectives of these meetings are to help select the dosing regimens for the next stage (typically Phases IIb and III) of drug development and to design informative dose-response trials that, by effectively incorporating prior quantitative knowledge, will improve the design of later-stage clinical trials. Furthermore, the sponsor might also seek the advice of the FDA on potential novel aspects of trial design, such as the use of adaptive designs, Bayesian statistical analyses and pharmacogenetic information.

As well as the value of gaining a regulatory perspective at an early stage on these novel design possibilities, which have the potential to substantially enhance the effectiveness of clinical development strategies (discussed in REF. 4), these meetings could help sponsors to tap into the unique wealth of data held by the FDA on previous drug development programmes. Although the FDA cannot share patient-level data without consent from the sponsors, key information such as models can be extracted from the data - for example, describing disease progression rate, placebo effect, patient dropout rate and the relationship of biomarkers to primary disease end points. If appropriately resourced and implemented, early interactions between the FDA and sponsors could therefore play an important part in tackling the rising costs and crippling failure rates in late-stage clinical development.

\footnotetext{
1. Booz Allen Hamilton. Independent evaluation of the Food and Drug Adminstration's first cycle review performance - retrospective analysis final report. The FDA website [online] < http://www.fda.gov/OHRMS/ DOCKETS/98fr/oc05257-rpt0001.pdf > (2006).

2. Wang, Y. et al. Leveraging prior quantitative knowledge to guide drug development decisions and regulatory science recommendations: impact of FDA pharmacometrics during 2004-2006. J. Clin. Pharmacol. 48, 146-156 (2008).

3. US FDA. Guidance for industry. End-of-Phase 2a meetings. The FDA website [online] < http://www.fda.gov/downloads/Drugs/ GuidanceComplianceRegulatoryInformation/Guidances/ucm079690. pdf $>$ (2009)

4. Orloff, J. et al. The future of drug development: advancing clinical trial design. Nature Rev. Drug Discov. 9 Oct 2009 (doi:10.1038/ nrd3025).
} 